\title{
Untersuchungen an Diazoverbindungen
}

V. Mitteilung*: Eine kinetische Methode zur Prüfung der Fragc, ob die unter Stickstoffentwicklung verlaufende, thermische Umsetzung einer Diazoverbindung als ,Diazo“- oder "Carben-Reaktion“ abläuft"1

Egon Fahr, Karl-heinz Keil, Hanns Lind und Franz Scheckenbach

Chemisches Institut der Universität Würzburg

(Z. Naturforschg. 20 b, 526-536 [1965] ; eingegangen am 3. Februar 1965)

\begin{abstract}
An Hand eines größeren Untersuchungsmaterials (Umsetzungen von Diazoverbindungen mit $a$-Carbonyl- und $a, \alpha^{\prime}$-Dicarbonyl-azo-Verbindungen, aromatischen Azoverbindungen, Nitrosoverbindungen, $\mathrm{S}$ c h if f schen Basen, Cyanessigsäure-äthylester, $p$-Tolunitril und Nitromethan) konnte gezeigt werden, daß sich mittels einer einfachen kinetischen Methode [Vergleich der Geschwindigkeit der Stickstoffentwicklung der Umsetzung mit der der thermischen Zersetzung der Diazoverbindung allein („Eigenzerfall“) ] entscheiden läßt, ob die unter Stickstoffentwicklung verlaufende, thermische Umsetzung einer Diazoverbindung als „Diazo-Reaktion“ (Reaktion der Diazoverbindung selbst) oder als „Carben-Reaktion“ (primäre Bildung des entsprechenden Carbens) abläuft.
\end{abstract}

Die Vielzahl der unter Stickstoffentwicklung ablaufenden thermischen Reaktionen der Diazoverbindungen ${ }^{2}$ lassen sich in zwei Gruppen unterteilen: In solche, bei denen die Diazoverbindungen unmittelbar mit dem Reaktionspartner in Reaktion treten („Diazo-Reaktion“) ; in solche, bei denen primär aus der Diazoverbindung das entsprechende Carben gebildet wird ("Carben-Reaktion“), das sich dann entweder als solches mit dem Reaktionspartner umsetzt oder Secundär-Reaktionen zeigt.

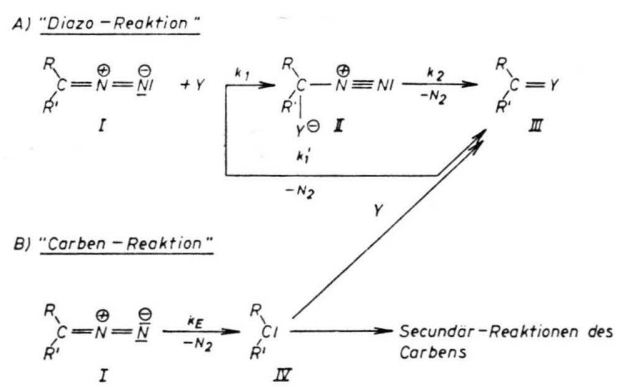

Schematisch ${ }^{3}$ dargestellt geht beim Reaktions-

* I. Mitt.: E. FAHR, Chem. Ber. 92, 398 [1959]; II. Mitt.: E. FAHR, Liebigs Ann. Chem. 627, 213 [1959]; III. Mitt.: E. F $\mathrm{AHR}$, Liebigs Ann. Chem. 638, 1 [1960] ; IV. Mitt.: E. $\mathrm{F}_{\mathrm{AHR}}$ u. W. D. Hörmann, Liebigs Ann. Chem. 682, 48 [1965].

1 Die Arbeit enthält Teile folgender Dissertationen (alle Univ. Würzburg) : a) K. H. KEIL, 1963; b) H. Lind, 1964; c) F. Scheckenbach, 1961.

2 Vgl. dazu die Úbersicht von R. Huisgen, Angew. Chem. 67, 439 [1955]. ablauf als „Diazo-Reaktion“ die Diazoverbindung I beim elektrophilen Angriff des Reaktionspartners Y entweder in den Zwischenstoff II über, der dann in das Endprodukt der Reaktion III zerfällt, oder liefert unter synchron ablaufender Stickstoffabspaltung direkt III. Beim Ablauf als "Carben-Reaktion“ geht I dagegen in das Carben IV über, das nun entweder mit dem Reaktionspartner Y III gibt oder Secundär-Reaktionen des Carbens zeigt.

Anders liegen die Verhältnisse bei der Umsetzung einer $a$-Carbonyl-diazo-Verbindung. Hier führt die „Diazo-Reaktion“ entweder über den Zwischenstoff II a oder in synchronem Ablauf zum Endprodukt III a. Dagegen entsteht bei der "Carben-Reaktion“" das $\alpha$-Keto-carben IV a, das im allgemeinen nicht als solches mit $\mathrm{Y}$ reagiert, sondern sich unter $\mathrm{W}$ olf $\mathrm{f}$ scher Umlagerung in das Keten V umlagert ${ }^{4}$. V setzt sich dann in Abhängigkeit von der Natur des Reaktionspartners $\mathrm{Y}$ entweder mit diesem z.B. zu VI oder VII um oder zeigt, ohne mit $\mathrm{Y}$ zu reagieren, Secundär-Reaktionen wie Dimerisation, Polymerisation und ähnliches.

${ }^{3}$ Die von uns gewählte vereinfachende schematische Darstellung genügt, wie im folgenden gezeigt wird, völlig für die Anwendung der kinetischen Methode.

${ }^{4}$ Zur Frage des Verhaltens von $a$-Keto-carbenen s. die eingehende Diskussion bei: a) R. Huisgen, H. König, G. Binsch u. H. J. Sturm, Angew. Chem. 73, 368 [1961]; b) R. Huisgen, G. Binsch u. L. Ghosez, Chem. Ber. 97, 2628 [1964] ; c) R. Huisgen, H. J. Sturm u. G. Binsch, 97, 2864 [1964]; d) R. Huisgen, G. Binsch u. H. König, 97, 2868, 2884 [1964]; e) G. Binsch, R. Huisgen u. H. KöNIG, 97, 2893 [1964]. 


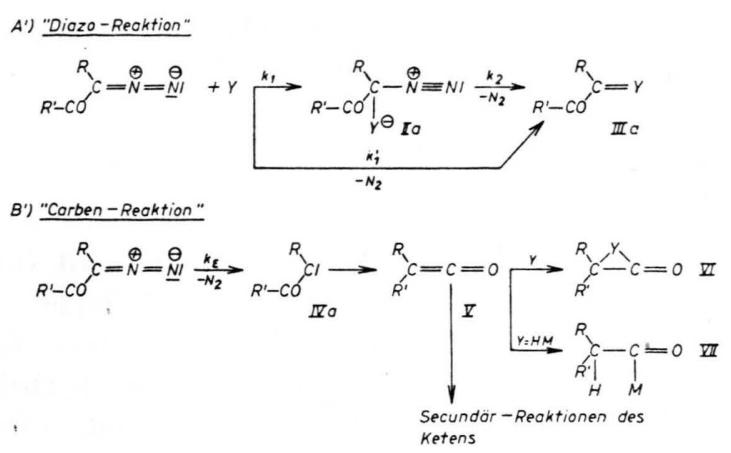

Das Verhalten der aus $\alpha$-Carbonyl- und $\alpha, \alpha^{\prime}$-Dicarbonyl-diazo-Verbindungen

$$
\left(\mathrm{R}-\mathrm{CO}-\mathrm{CN}_{2}-\mathrm{R}^{\prime}, \quad \mathrm{R}-\mathrm{CO}-\mathrm{CN}_{2}-\mathrm{CO}-\mathrm{R}^{\prime}\right)
$$

in Freiheit gesetzten $\alpha$-Keto-carbene ist von der Natur der Reste $R$ und $\mathrm{R}^{\prime}$ sowie von den Reaktionsbedingungen abhängig. Während Azibenzil und die $\alpha, \alpha^{\prime}$-Dicarbonyl-diazo-Verbindungen im allgemeinen ausschließlich in die entsprechenden Ketene übergehen, isomerisieren sich die bei der Zersetzung von $\alpha$-Carbonyl-diazoVerbindungen der Struktur $\mathrm{R}-\mathrm{CO}-\mathrm{CN}_{2}-\mathrm{CH}_{2}-\mathrm{R}^{\prime}$ entstehenden $\alpha$-Keto-carbene bei niedriger Temperatur $\mathrm{zu}$ den Olefinen $\mathrm{R}-\mathrm{CO}-\mathrm{CH}=\mathrm{CH}-\mathrm{R}^{\prime}$, bei höherer Temperatur erfolgt Wolff sche Umlagerung zu den Ketenen ${ }^{5 a-c}$.

Die $\alpha$-Keto-carbene der Diazoessigsäure- und Diazomalonsäureester sowie einiger Perfluor- und Perchlorcarbonyl-diazo-Verbindungen lagern sich nicht zu den Ketenen um; sie reagieren als solche weiter ${ }^{6,7}$.

Während nun die Feststellung, ob das Reaktionsprodukt III in einer "Diazo-Reaktion“ oder "Carben-Reaktion“ gebildet wird, nur für die Kenntnis der Reaktionsmechanismen nicht aber für die präparative organische Chemie von Bedeutung ist, liefern dagegen bei der Umsetzung einer zum Keten umlagerbaren $\alpha$-Carbonyl-diazo-Verbindung „Diazo-

5 a) V. Franzen, Liebigs Ann. Chem. 602, 199 [1957]; b) Vgl. dazu auch die von P. YAtes u. T. J. Clark, Tetrahedron Letters [London] 1961, 435, bei der thermischen Zersetzung des Benzoyl-diazomethans erhaltenen Produkte sowie Lit. $4 \mathrm{a}-$ e. c) Erfolgt die thermische Zersetzung der a-Carbonyl-diazo-Verbindung in Gegenwart von Metallen oder deren Salze, so kommt es in vielen Fällen zu CarbenReaktionen ${ }^{9}$; vgl. dazu J. Novak, J. Ratusky, V. SNeberg u. F. Sorm, Chem. listy 51, 479 [1957] (C. A. 51, 10508 [1957]), J. Ratusky u. F. Sorm, Chem. listy 51, 1091 [1957] (C. A. 51, 13843 [1957]), I. Ernest u. H. JelinKova, Collect. czechoslov. chem. Commun. 24, 3341 [1959]

(C. A. 54, 4375 [1960]), H. Strzelecka u. M. Siemiatycki, C. R. hebd. Séances Acad. Sci. 252, 3821 [1961] (C. A. 55, 22209 [1961]) sowie P. Yates, J. Amer. chem. Soc. 74, 5376 [1952].

${ }^{6}$ F. Weygand, W. Schwenke u. H. J. Bestmann, Angew. Chem. 70, 506 [1958]; F. Weygand, H. Dworschak, K. Koch u. S. Konstas, Angew. Chem. 73, 409 [1961]; F. Weygand u. K. Koch, Angew. Chem. 73, 531 [1961].
Reaktion" und "Carben-Reaktion" verschiedene Produkte; d.h. also, daß die Entscheidung, ob die Umsetzung einer $\alpha$-Carbonyl-diazo-Verbindung mit einem bestimmten Reaktionspartner als „Diazo“. oder "Carben-Reaktion“ abläuft, eine Voraussage auf die Konstitution des sich bei dieser Umsetzung bildenden Produktes erlaubt und damit für die präparative organische Chemie von Wert ist.

Um auf einfachem kinetischen Wege unterscheiden zu können, ob die Umsetzung als „Diazo-“ oder "Carben-Reaktion“ abläuft, wurden von uns folgende Annahmen gemacht:

a) Der Ablauf als „Diazo-Reaktion“ erfolgt nach II. Ordnung (entweder synchroner Ablauf oder $k_{2}>k_{1}$ ).

b) Der Reaktionspartner Y begünstigt beim Ablauf als "Carben-Reaktion" die Bildung des Carbens bzw. $\alpha$-Keto-carbens nicht. Damit wird die Geschwindigkeit der Stickstoffentwicklung der Umsetzung gleich der des Eigenzerfalls ${ }^{8}$ der Diazoverbindung sein und nach I. Ordnung ablaufen ${ }^{9}$.

c) Beim Ablauf als „Diazo-Reaktion“ wird die Diazogruppierung durch den elektrophilen Angriff von $\mathrm{Y}$ aktiviert ${ }^{10}$; d. h. die Stickstoffentwicklung der Umsetzung verläuft schneller als der Eigenzerfall der Diazoverbindung.

Für die kinetische Messung durch Bestimmung der Stickstoffentwicklung war allerdings zu erwarten, daß der Ablauf im strengen, von den Mechanismen $\mathrm{A}, \mathrm{B}, \mathrm{A}^{\prime}$ und $\mathrm{B}^{\prime}$ geforderten Sinne nur relativ selten auftreten wird; und zwar für die Mechanismen A und $\mathrm{A}^{\prime}$ nur dann, wenn die "Aktivierung“ der Diazogruppierung durch den Reaktionspartner

7 A. Roedig, E. Fahr u. H. Aman, Chem. Ber. 97, 77 [1964] ; A. Roedig, H. Aman u. E. F $\mathrm{AHR}$, Liebigs Ann. Chem. 675, 47 [1964].

8 Mit Eigenzerfall ist die Geschwindigkeit der Stickstoffentwicklung des Zerfalls der Diazoverbindung in Abwesenheit von Reaktionspartnern bezeichnet.

9 Der Eigenzerfall von Diazoverbindungen wird durch Metalle, Metallsalze, rauhe Oberflächen (Glasschliffe, rauhe oder angeritzte Glasoberflächen, Siedesteinchen u. ä.) sowie mechanische Verunreinigungen $\mathrm{z}$. T. grundlegend verändert. Die Anwendung der hier beschriebenen Methode setzt deshalb deren Abwesenheit voraus (vgl. dazu auch die Ausführungen auf S. 529). So dürfte der kürzlich von H. Goetz u. H. Juds, Liebigs Ann. Chem. 678, 1 [1964] beobachtete Zerfall des Diazofluorens nach II. Ordnung vermutlich durch die Metallteile der Meßzelle ausgelöst worden sein.

10 Unter „Aktivierung“ der Diazogruppierung sei die durch den Angriff des Reaktionspartners Y bewirkte Lockerung der $\mathbf{C}-\mathrm{N}_{2}$-Bindung verstanden. 
so stark ist, daß der Eigenzerfall der Diazoverbindung bei der Umsetzungstemperatur praktisch gleich null ist.

So erfolgt die Umsetzung der Diazoalkane und Diazoketone mit anorganischen Säuren unter sehr starker „Aktivierung“ der Diazogruppe. Sie verläuft deshalb schon bei Raumtemperatur, bei der diese Verbindungen nur einen sehr kleinen Eigenzerfall haben, sehr schnell.

Ist dagegen die durch den Reaktionspartner ausgelöste „Aktivierung“ der Diazogruppierung geringer, so wird zur Ausführung der Reaktion eine entsprechend höhere Temperatur nötig sein. Das bedeutet, daß in mehr oder weniger ausgeprägtem $\mathrm{Maße}$ der Eigenzerfall der Diazoverbindung mit dem Ablauf nach A bzw. A' konkurrieren wird. Als Konkurrenz-Reaktionen kommen dabei in Frage:

a) Der Zerfall der Diazoverbindung zum ÄthylenDerivat;

b) Die Umsetzung des Carbens mit der Diazoverbindung zum Ketazin.

Eine a entsprechende Störung bewirkt als Zweigreaktion ein Absinken der Konzentration der Diazoverbindung; b verursacht durch Fixierung eines Teils der eingesetzten Diazoverbindung einen Fehlbetrag an gasförmigem Stickstoff. Um diese Zweigreaktionen von der Hauptreaktion zu eliminieren und damit exakte kinetische Angaben zu erhalten, wäre es notwendig, nicht nur die Geschwindigkeit der Stickstoffentwicklung, sondern auch die Konzentrations-Abnahme der eingesetzten Diazoverbindung und die des Reaktionspartners zu bestimmen. Wie im folgenden gezeigt wird, genügt im allgemeinen jedoch ein Vergleich des Eigenzerfalls der Diazoverbindung und der Geschwindigkeit der Stickstoffentwicklung der Umsetzung bei gleicher Temperatur und im gleichen Lösungsmittel, um ein präparativ verwertbares Ergebnis zu erhalten.

Außer der Umsetzung nach den Mechanismen A, $\mathrm{A}^{\prime}$ und $\mathrm{B}, \mathrm{B}^{\prime}$ besteht noch die Möglichkeit, daß die Diazoverbindung mit dem Reaktionspartner $\mathrm{Y}$ die cyclische Verbindung VIII bildet, die nun unter den Reaktionsbedingungen Stickstoff abspaltet und so

11 Ein dem Mechanismus C entsprechender Reaktionsablauf ist sehr eingehend von L. Horner u. E. Lingnau ${ }^{12}$ diskutiert und an Hand der Umsetzung von Olefinen mit Diazofluoren kinetisch untersucht worden.

12 L. Horner u. E. Lingnau, Liebigs Ann. Chem. 591, 21 [1955].

13 Siehe dazu die Umsetzung von Diazofluoren mit Benzilidenanilin auf S. 534 . eine unter Stickstoffabspaltung ablaufende Reaktion der Diazoverbindung selbst vortäuscht.

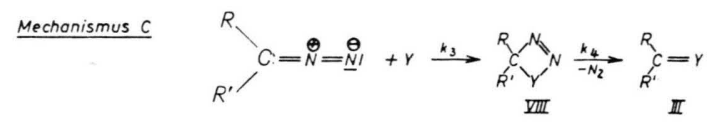

Das Vorliegen von Mechanismus $\mathrm{C}^{11}$ wird vor allem daran zu erkennen sein, daß die Reaktion bedingt durch $k_{4}$ - eine vom Eigenzerfall der Diazoverbindung verschiedene, aber nach der I. Ordnung ablaufende Stickstoffentwicklung zeigt, während beim Ablauf nach A bzw. $\mathrm{A}^{\prime}$ mit $k_{2}>k_{1}$ bzw. synchronem Mechanismus die Stickstoffentwicklung der Umsetzung nach II. Ordnung erfolgt. Unter den von uns bis jetzt ausgeführten Messungen befindet sich nur ein Fall, bei dem vermutlich ein Ablauf nach Mechanismus $\mathrm{C}$ vorliegt ${ }^{13}$.

In der vorliegenden Arbeit haben wir an einem größeren Substanzmaterial durch gasvolumetrische Messungen den Eigenzerfall einer Diazoverbindung mit der Geschwindigkeit der Stickstoffentwicklung ihrer Umsetzung mit einem Reaktionspartner verglichen und geprüft, wieweit sich auf diesem Wege die obigen Annahmen bestätigen lassen ${ }^{14}$. Als Reaktionspartner wurden Vertreter folgender Substanzklassen ausgewählt: $\alpha$-Carbonyl- und $\alpha, \alpha^{\prime}$-Dicarbonyl-azo-Verbindungen, aromatische Azoverbindungen, Nitrosoverbindungen, $\mathrm{S} \mathrm{ch}$ if $\mathrm{f}$ sche Basen sowie Cyanessigsäure-äthylester, $p$-Tolunitril und $\mathrm{Ni}$ tromethan.

\section{Ausführung der kinetischen Messungen}

Die Umsetzung erfolgt im Reaktionsgefäß 1 (Volumen der Kugel ca. $50 \mathrm{ml})^{15}$, das in ein thermostatisiertes Heizbad (Ölthermostat) 2 eintaucht. In das Reaktionsgefäß wird diejenige der Reaktionskomponenten gegeben, die bei der gewählten Reaktionstemperatur thermisch am beständigsten ist, und in $10 \mathrm{ml}$ des Lösungsmittels ${ }^{16}$ gelöst. Das Einwurfgefäß 3 enthält die zweite Reaktionskomponente. Löst sich diese bei der Reaktionstemperatur im verwandten Lösungsmittel nur langsam, so empfiehlt es sich, die Substanz bereits gelöst in das dann dementsprechend größer zu dimensionierende Einwurfgefäß zu geben. Danach wird das Reaktionsgefäß mit Stickstoff gespült, über das Kapil-

14 Kürzlich ist von R. Hursgen u. Mitarbb. ${ }^{4} \mathrm{c}-\mathrm{e}$ der analoge Vergleich von Eigenzerfall und Stickstoffentwicklung der Umsetzung zum Nachweis des aus Tetrachlorbenzol-odiaz-oxid entstehenden $a$-Keto-carbens angewandt worden.

15 Die oben beschriebene Apparatur ist auf Umsetzungen mit 4 mMol Substanz dimensioniert.

16 Von uns wurde im allgemeinen absolutes Xylol verwandt. 
larrohr $4^{16 a}$ an die thermostatisierte Gasbürette 5 angeschlossen und mit Hilfe des Exzenters 6 intensiv geschüttelt ${ }^{17}$. Nach einigen Min. wird durch Auslösen der Fallmechanik 7 (Herausziehen des glasummantelten Eisenkerns mittels eines Magneten) die im Einwurfgefäß befindliche Substanz in das Reaktionsgefäß gegeben.

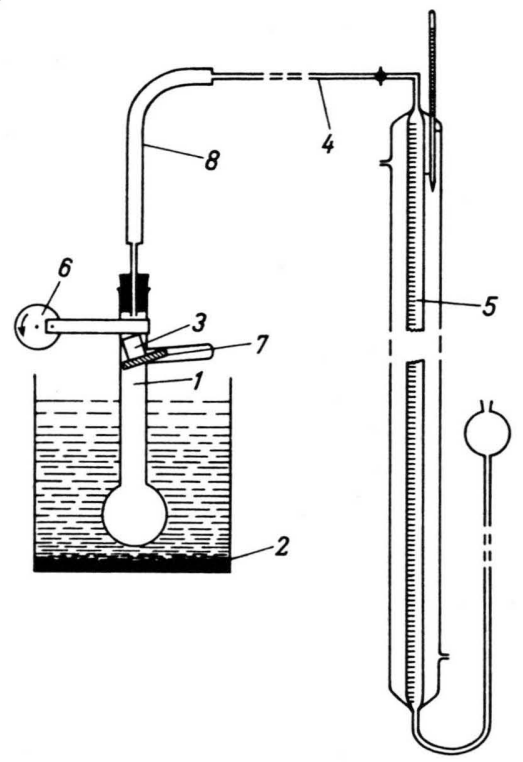

Abb. 1. Apparatur zur Messung der Gasentwicklung.

Analog erfolgt die Bestimmung des Eigenzerfalls. Hier wird das Lösungsmittel in 1 vorgelegt und die Diazoverbindung in das Einwurfgefäß 3 gegeben.

Um eine präparativ verwertbare Aussage zu erhalten, ist es nicht nötig, die ReaktionsgeschwindigkeitsKonstante $(R G K)$ der Umsetzung bzw. des Eigenzerfalls aus den Daten der Stickstoffentwicklung zu bestimmen. Es genügt völlig, das entwickelte Stickstoffvolumen der Umsetzung gegen die Zeit aufzutragen und mit dem Eigenzerfall der Diazoverbindung zu vergleichen (s. dazu die Abbn. 3 bis 7).

In den Fällen, in denen von uns $R G K$-Werte in den
Tabn. 1 bis 5 angegeben worden sind, erfolgte deren Bestimmung unter folgenden Bedingungen: Temperatur-Genauigkeit im Reaktionsbad $\pm 1^{\circ}$, in der Gasbürette $\pm 0,5^{\circ}$; bei Reaktionen II. Ordnung wurde das Volumen der Reaktionslösung bei der Umsetzungstemperatur in einem getrennten Ansatz durch Volumenmessung bestimmt.

Während sich die $R G K$ der als „Diazo-Reaktionen“ nach Mechanismus A bzw. A' ablaufenden Reaktionen recht genau bestimmen lassen, macht eine zuverlässige Angabe der $R G K$ des Eigenzerfalls Schwierigkeiten; und zwar scheint nach unseren Erfahrungen der Eigenzerfall der Diazoverbindungen $u$. a. von der Reinheit der Substanz und des Lösungsmittels abhängig zu sein. So erhielten wir für den Eigenzerfall des Diazofluorens $\left(80^{\circ}\right.$, Xylol) bei sechs verschiedenen Bestimmungen Werte zwischen $2,0 \cdot 10^{-4}$ und $4,1 \cdot 10^{-4}\left[\mathrm{~min}^{-1}\right]$. Die $R G K$ des Eigenzerfalls ist deshalb von uns nur größenordnungsmäßig angegeben worden ${ }^{18}$.

Bei einigen Umsetzungen (s. Tabn. 3 und 4) wird außer Stickstoff auch Kohlendioxyd entwickelt. Um hier den Eigenzerfall und die Stickstoffentwicklung der Umsetzung miteinander vergleichen zu können, muß die $\mathrm{CO}_{2}$-Entwicklung eliminiert werden. Das kann nach folgender Methode geschehen:

In das Reaktionsgefäß 1 (Abb. 2) ${ }^{16 a}$, das mit einem seitlichen Gaseinleitungsrohr versehen ist, wird die xylolische Lösung der thermisch stabilsten Substanz gegeben und im thermostatisierten Reaktionsbad 2 mit Hilfe des Exzenters 3 geschüttelt. Das Gaseinleitungsrohr ist so einzuführen, daß es gerade über der Flüssigkeitsoberfläche zu stehen kommt. Bei geöffneten Hähnen 6 reguliert man den Stickstoffstrom ${ }^{19}$ aus der Bombe mittels des Blasenzählers 10 (gefüllt mit 50proz. $\mathrm{KOH}$ ) auf einen Gasdurchsatz von ca. $6 \mathrm{Bla-}$ sen/Sek. ein und erhält die Apparatur nach ca. 15 Min. luftfrei. Danach wird die Reaktion durch Betätigung des Fallmechanismus ausgelöst und die $\mathrm{CO}_{2}$-Bestimmung durch abwechselndes Beladen und Auswägen der Absorptionsrohre 7 (in Richtung des Gasdurchganges gefüllt mit Watte, Natronasbest, Watte, feinkörnigem Calciumchlorid, Watte) ausgeführt. Das U-Rohr 8 (gefüllt mit Natronasbest und Calciumchlorid) schließt die Apparatur nach außen ab.

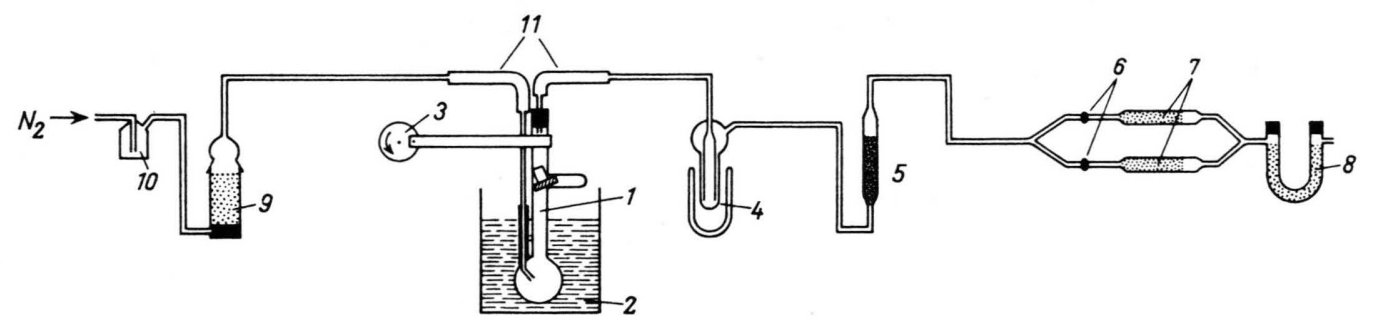

Abb. 2. Apparatur zur Messung der Kohlendioxyd-Entwicklung.

16a Die Mikro-Vakuumschläuche 8 (Abb. 1) bzw. 11 (Abb. 2) dienen zur elastischen Verbindung von Reaktionsgefä $\beta$ und Apparatur.

17 Exzenterhub zwischen 5 und $10 \mathrm{~mm}$ bei 100 bis 300 MotorUmdrehungen/Minute.
18 Die oben erwähnte Beeinflussung des Eigenzerfalls ist jedoch so klein, daß sie die Anwendbarkeit der in der vorliegenden Arbeit beschriebenen Methode nicht beeinflußt.

19 Gereinigt im Trockenturm 9 (aufeinanderfolgende Schichtten von Glaswolle, $\mathrm{P}_{2} \mathrm{O}_{5}$, Glaswolle, Natronasbest und Glaswolle). 
Schwierig ist es, das durch den Gasstrom mitgeschleppte Lösungsmittel zu entfernen. Wir absorbierten den weitaus größten Teil mit Hilfe der sich an das Reaktionsgefäß anschließenden, mit Eis-Kochsalz-Mischung gekühlten Kühlfalle 4 und des mit Blaugel ${ }^{20}$ gefüllten Trockenrohres 5. Da trotzdem immer noch geringe Lösungsmittelmengen in die Absorptionsrohre 7 gelangen und dadurch eine Verfälschung der Kohlendioxyd-Werte verursachen, wurden mit der Strömungsgeschwindigkeit und Badtemperatur der Umsetzung diese Blindwerte bestimmt und damit die gemessenen Werte korrigiert ${ }^{21}$. Da das Kohlendioxyd nicht im Augenblick seines Entstehens, sondern in Abhängigkeit von der Größe der Apparatur und der Strömungsgeschwindigkeit des Gases in 7 absorbiert wird, ist das Verhältnis von Geschwindigkeit der Kohlendioxyd-Entwicklung/Strömungsgeschwindigkeit des Gases so zu wählen, daß letztere groß gegenüber der ersteren ist.

Um zu prüfen, ob auf diese Weise der „Verschleppungsfaktor" zuverlässig beseitigt werden kann, haben wir den zeitlichen Ablauf verschiedener Reaktionen, die ausschließlich Kohlendioxyd liefern, sowohl nach der unmittelbar ansprechenden gasvolumetrischen als auch nach der hier angegebenen gravimetrischen $\mathrm{Me}$ thode bestimmt. Wir erhielten übereinstimmende Ergebnisse ${ }^{21}$.

Die gewünschte Kurve der Stickstoffentwicklung erhält man, indem man die so erhaltenen $\mathrm{CO}_{2}$-Werte von der volumetrisch gemessenen Gesamtgas-Entwicklung $\left(\mathrm{N}_{2}+\mathrm{CO}_{2}\right)$ abzieht. Abb. 6 zeigt eine derartige Messung.

\section{Die Umsetzung von Diazoverbindungen mit Azoverbindungen ${ }^{22}$}

Aus den Tabn. 1 und 2 geht hervor, daß die Umsetzung von Diazofluoren, Diazoessigsäureester und Diazoketonen mit Azodicarbonsäureester sowie Azodibenzoyl als „Diazo-Reaktion“ erfolgt. Den Ablauf der Reaktion zeigen die Abbn. 3 und 5. Die Kurvenbilder der anderen Umsetzungen sind analog. Die Reaktion muß also als elektrophiler Angriff der $\alpha, \alpha^{\prime}$-Dicarbonyl-azo-Verbindung an der Diazoverbindung ablaufen. Wir vermuten, daß dabei das Diazonium-Ion IX durchlaufen wird, das dann in $\mathrm{X}$ zerfällt.

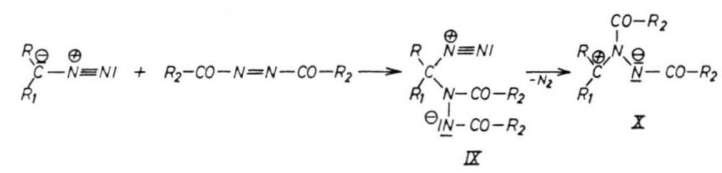

${ }^{20}$ Durch Blindversuche ist zu prüfen, ob das Blaugel $\mathrm{CO}_{2}$ absorbiert.

21 Siehe dazu die Ausführungen in Lit. l. c. ${ }^{1 b}$ und zwar auf S. 75 und 76.

22 Die präparativen Ergebnisse dieser Umsetzungen sowie die Untersuchung der Struktur der dabei entstehenden Produkte werden an anderer Stelle veröffentlicht; s. dazu die
Die Stabilisierung von $\mathrm{X}$ ist von den Reaktionsbedingungen und der Natur der Reste $R, R_{1}$ und $R_{2}$ abhängig ${ }^{22}$. Sie führt bei der Umsetzung von Diazofluoren und Diazoessigsäureester mit Azodicarbonsäureestern bei Temperaturen unter $80-100^{\circ}$ zu den Oxdiazolinen XI, von denen die aus Diazofluoren und Azodicarbonsäureester dargestellten in Lösung mit der dazu isomeren Azomethin-imin-Form XII im Gleichgewicht stehen ${ }^{22 a, b, e}$. Bei Temperaturen oberhalb $100^{\circ}$ erfolgt dagegen die Bildung der Hydrazone XIII.
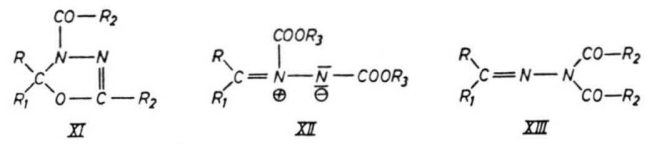

Die Mono-carbonyl-azo-Verbindungen Phenylazocarbonsäure-äthylester und Phenylazocarbonamid reagieren dagegen auch mit Diazofluoren nicht mehr. Analog verhält sich Azobenzol und $\omega, \omega^{\prime}$-Azotoluol. In allen diesen Fällen ist die Kurve der Stickstoffentwicklung der Umsetzung innerhalb der Fehlergrenze der Bestimmung mit der des Eigenzerfalls identisch. Hier erfolgt also keine Reaktion der Azoverbindung mit der Diazoverbindung selbst, sondern - wenn überhaupt - eine Umsetzung der Azoverbindung mit Produkten des thermischen Zerfalls der Diazoverbindung.

Analog verläuft die Umsetzung von Diazoacetessigsäure-äthylester, Benzoyl-diazoessigsäure-methylester, Diazodimedon, Benzoyl-diazoaceton und Azibenzil. Auch hier geht, wie der Vergleich von Eigenzerfall und Stickstoffentwicklung der Reaktion zeigt (s. Abb. 4), keine Reaktion zwischen der Azoverbindung und der $\alpha$-Carbonyl-diazo-Verbindung vor sich. Für den Reaktionsablauf ist damit zu erwarten, daß es nicht wie bei Umsetzung von Diazoessigsäureester und Diazoketonen zur Bildung von Oxdiazolin- bzw. Diacylhydrazon-Derivaten (entsprechend XI bzw. XIII) kommt, sondern daß entweder das aus der $\alpha$-Carbonyl-diazo-Verbindung durch Umlagerung des $\alpha$-Keto-carbens gebildete Keten mit der Azoverbindung reagiert oder nur Secundär-Reaktionen wie Dimerisation oder Verharzung zeigt. Die Aufarbeitung der Ansätze ergab, daß die Umsetzung von Azibenzil und Benzoyl-di-

Dissertationen (Lit. l. c. $\left.{ }^{1 \mathrm{a}} \mathrm{u} . \mathrm{b}\right)$, die Diplomarbeiten a) K. Döppert, 1962, b) K. Königsdorfer, 1964 und c) A. Jung, 1963 (alle Univ. Würzburg) sowie d) E. FAHR u. F. Scheckenbach, Liebigs Ann. Chem. 655, 86 [1962], e) E. Fahr, K. Döppert u. F. Scheckenbach, Angew. Chem. 75, 670 [1963], f) E. FAHr, Angew. Chem. 73, 536 [1961], ebenda 76, 505, 579 [1964]. 
azoessigsäure-methylester mit Azodicarbonsäure-diäthylester zu den Addukten der entsprechenden Ketene an die Azoverbindung führt ${ }^{23}$, während bei der Umsetzung von Diazoacetessigsäure-äthylester, Diazodimedon und Benzoyl-diazoaceton keine Addition des Azodicarbonsäureesters an die Ketene erfolgt ${ }^{24}$; erhalten wurde neben nicht umgesetztem Azodicarbonsäureester beim Diazodimedon das Dimere des Ketens XIV, beim Diazoacetessigsäureester und Benzoyl-diazoaceton ein stickstofffreies zähes Öl.
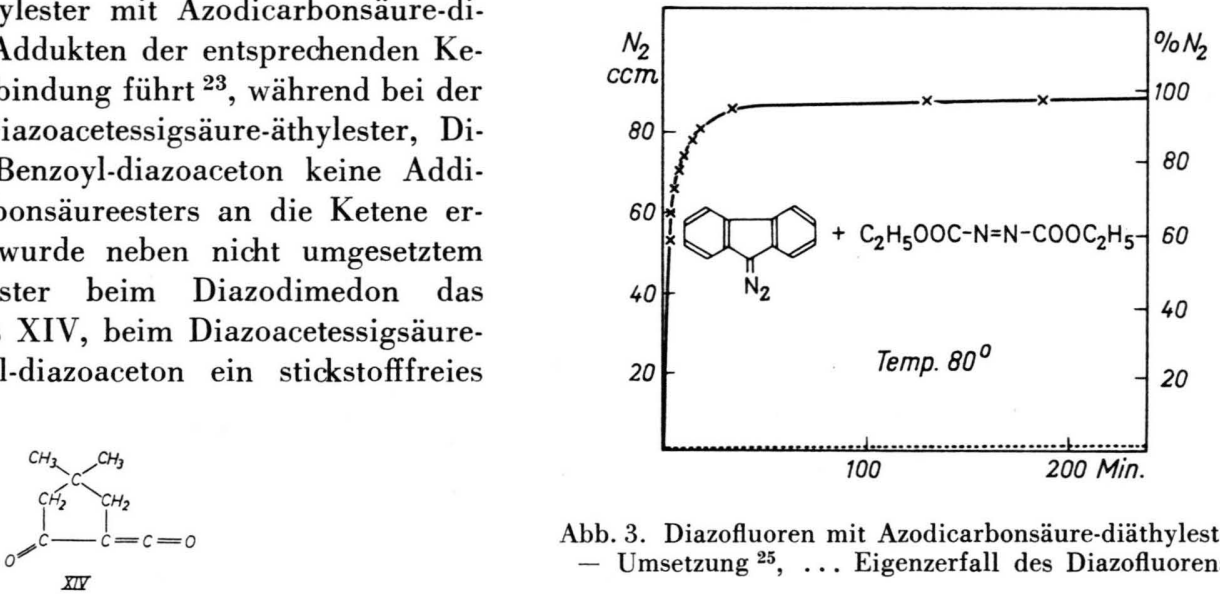

Abb. 3. Diazofluoren mit Azodicarbonsäure-diäthylester, - Umsetzung ${ }^{25}$, ... Eigenzerfall des Diazofluorens.

\begin{tabular}{|c|c|c|c|c|c|c|}
\hline \multirow{2}{*}{ Diazoverbindungen } & \multirow{2}{*}{ Azoverbindung } & \multirow{2}{*}{$\begin{array}{c}\text { Reaktions- } \\
\text { Temperatur } \\
{\left[{ }^{\circ} \mathrm{C}\right]}\end{array}$} & \multirow{2}{*}{$\begin{array}{l}\text { Vergleich } \\
\text { von Eigen- } \\
\text { zerfall und } \\
\mathrm{N}_{2} \text {-Entwick- } \\
\text { lung der } \\
\text { Reaktion }{ }^{26}\end{array}$} & \multicolumn{2}{|c|}{ RGK der Reaktion } & \multirow{2}{*}{$\begin{array}{c}\text { RGK des } \\
\text { Eigen- } \\
\text { zerfalls } \\
{\left[\mathrm{min}^{-1}\right]}\end{array}$} \\
\hline & & & & $\begin{array}{l}{\left[\mathrm{min}^{-1}\right.} \\
\left.\cdot \mathrm{mol}^{-1}\right]\end{array}$ & {$\left[\min ^{-1}\right]$} & \\
\hline Diazofluoren & $\begin{array}{l}\text { Azodicarbonsäure- } \\
\text { diäthylester } 27\end{array}$ & 80 & $(+)$ Abb. 3 & 1,28 & & $2 \cdot 10^{-428}$ \\
\hline , & $\begin{array}{l}\text { Azodicarbonsäure- } \\
\text { dibenzylester }\end{array}$ & 60 & $(+)$ & 2,0 & & $0,5 \cdot 10^{-4}$ \\
\hline $\begin{array}{l}\text { Diazoessigsäure- } \\
\text { äthylester }\end{array}$ & $\begin{array}{l}\text { Azodicarbonsäure- } \\
\text { diäthylester }\end{array}$ & 80 & $(+)$ & 29 & & 29 \\
\hline Azibutanon &, & 80 & $(+)$ & 0,15 & & 29 \\
\hline Azibenzil & , & 80 & (-) Abb. 4 & & 0,2 & 0,2 \\
\hline $\begin{array}{l}\text { Diazoacetessigsäure- } \\
\text { äthylester }\end{array}$ & $"$ & 120 & $(-)$ & & 0,01 & 0,01 \\
\hline $\begin{array}{l}\text { Benzoyl-diazoessig- } \\
\text { säure-methylester }\end{array}$ & ," & 120 & $(-)$ & & 0,04 & 0,04 \\
\hline Diazodimedon & ,", & 120 & $(-)$ & & 0,05 & 0,05 \\
\hline Benzoyl-diazoaceton & , & 110 & $(-)$ & & 0,2 & 0,2 \\
\hline Diazofluoren & Azobenzol & 80 & $(-)$ & & $2 \cdot 10^{-4}$ & $2 \cdot 10^{-428}$ \\
\hline & $\begin{array}{l}\text { Phenylazocarbon- } \\
\text { säure-äthylester }\end{array}$ & 80 & $(-)$ & & $5 \cdot 10^{-4}$ & $2 \cdot 10^{-428}$ \\
\hline, & Phenylazocarbonamid & 80 & $(-)$ & & $5 \cdot 10^{-4}$ & $2 \cdot 10^{-428}$ \\
\hline , & Azodibenzoyl & $\begin{array}{r}60 \\
80\end{array}$ & $(+)$ & 0,77 & ?.10-4 & $0,5 \cdot 10^{-4}$ \\
\hline $\begin{array}{l}\text { o-Nitrobenzoyl-diazo- } \\
\text { methan }\end{array}$ & $\begin{array}{l}\omega . \omega \text {-Azotoluol } \\
\text { Azodibenzoyl }\end{array}$ & $\begin{array}{l}80 \\
80\end{array}$ & $(+)$ & 0,13 & & 29 \\
\hline
\end{tabular}

Tab. 1. Umsetzungen von Diazoverbindungen mit Azoverbindungen ${ }^{25}$.

23 Benzoyl-diazoessigsäure-methylester liefert mit Azodicarbonsäure-diäthylester das 1.2-Dicarbäthoxy-3-phenyl-3carbomethoxy-diazetidinon ${ }^{1} \mathrm{c}$; zur Struktur der aus Azibenzil und Azodicarbonsäure-diäthylester entstehenden komplizierten Substanzen siehe Lit. 1. c. ${ }^{1 \mathrm{a}}$

24 Analog verhalten sich die Umsetzungen von Propionyldiazoessigsäure-äthylester, Dibenzoyl-diazomethan und 2Diazo-3-ketobernsteinsäure-äthylester.

25 Wenn nicht anders angegeben, 4,0 $\mathrm{mMol}$ der Diazoverbindung und 4,0 mMol der Reaktionskomponente in 10,0 ml absol. Xylol gelöst.

$26(+)$ : Eigenzerfall und Stickstoffentwicklung der Umsetzung sind deutlich verschieden. (-): Eigenzerfall und Stickstoffentwicklung der Umsetzung sind innerhalb der Meßgenauigkeit identisch.
27 Die Umsetzung von Diazofluoren mit Azodicarbonsäure-diäthylester und Azodibenzoyl wurde bereits von L. HoRner u. E. Lingnau ${ }^{12}$ kinetisch untersucht. Sie diskutierten für die Umsetzung mit Azodicarbonsäure-diäthylester eine Reaktion, die anfangs nach I. Ordnung verläuft, dann durch eine Simultan-Reaktion verdrängt wird. Für die Umsetzung mit Azodibenzoyl erhielten sie keinen über einen längeren Zeitraum konstanten Wert für die $R G K$. Die wiederholt von uns ausgeführte Umsetzung von Diazofluoren mit Azodicarbonsäure-diäthylester zeigte einen eindeutigen Verlauf nach II. Ordnung mit der oben angegebenen $R G K$.

28 Zum Eigenzerfall des Diazofluorens s. die Ausführungen auf S. 529.

29 Die $R G K$ der Umsetzung bzw. des Eigenzerfalls konnte nicht zuverlässig bestimmt werden. 


\begin{tabular}{|c|c|c|}
\hline Diazoketon & $\begin{array}{l}\text { Vergleich von } \\
\text { Eigenzerfall } \\
\text { und } \mathrm{N}_{2} \text {-Ent- } \\
\text { wicklung der } \\
\text { Reaktion }{ }^{26}\end{array}$ & $\begin{array}{c}R G K \text { der } \\
\text { Reaktion } \\
{\left[\mathrm{min}^{-1}\right.} \\
\left.\cdot \mathrm{mol}^{-1}\right]\end{array}$ \\
\hline \multirow{8}{*}{$\begin{array}{l}\mathrm{CHN}_{2}-\mathrm{CO}-\mathrm{COOC}_{2} \mathrm{H}_{5} \\
p \text {-Nitro-benzoyl- } \\
\text { diazomethan } \\
p \text {-Brom-benzoyl- } \\
\text { diazomethan } \\
\alpha \text {-Naphthoyl- } \\
\text { diazomethan } \\
o \text {-Nitro-benzoyl- } \\
\text { diazomethan } \\
\text { Benzoyl-diazomethan } \\
p \text {-Methyl-benzoyl- } \\
\text { diazomethan } \\
\text { Phenyl-acetyl- } \\
\text { diazomethan }\end{array}$} & $(+)$ & 0,007 \\
\hline & $(+)$ & 0,039 \\
\hline & $(+)$ & 0,060 \\
\hline & $(+)$ & 0,063 \\
\hline & $(+)$ & 0,066 \\
\hline & $(+)$ Abb. 5 & 0,086 \\
\hline & $(+)$ & 0,12 \\
\hline & $(+)$ & 0,17 \\
\hline
\end{tabular}

Tab. 2. Umsetzung von Diazoketonen mit Azodicarbonsäurediäthylester bei $80^{\circ}$ in absol. Xylol ${ }^{25}$.

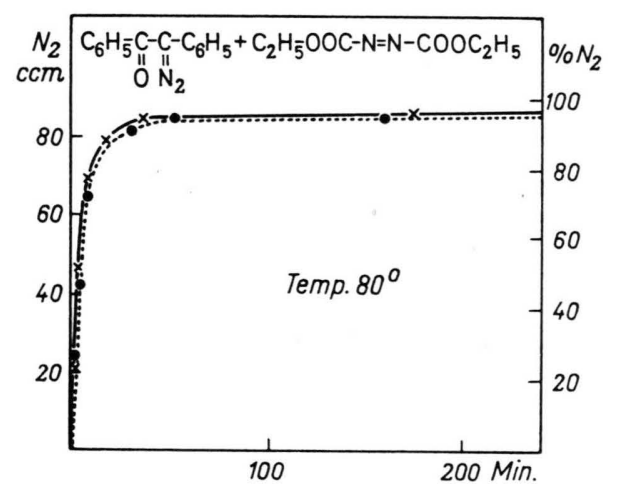

Abb. 4. Azibenzil mit Azodicarbonsäure-diäthylester, $-x-x$ - Umsetzung ${ }^{25}, \cdot \cdots \bullet$ Eigenzerfall des Azibenzils.

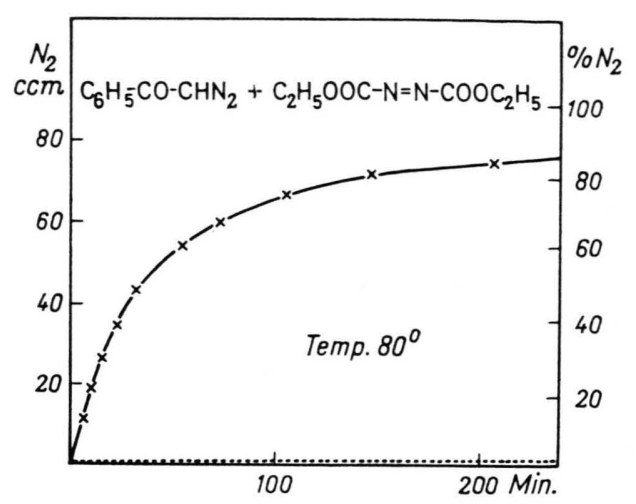

Abb. 5. Benzoyl-diazomethan mit Azodicarbonsäure-diäthylester, - Umsetzung ${ }^{25}$, .... Eigenzerfall des Benzoyl-diazomethans.

\section{Die Umsetzung von Diazoverbindungen mit Nitrosoverbindungen}

Aus der Tab. 3 geht hervor, daß die Umsetzung von Diazofluoren und Diazoessigsäure-äthylester mit Nitrosobenzol bzw. p-Nitroso-dimethylanilin als „Diazo-Reaktion“ abläuft. Die Kurvenbilder entsprechen der Abb. 3.

Als Mechanismus ist deshalb ein elektrophiler Angriff der Nitrosoverbindung anzunehmen, der entweder über die Diazonium-Verbindung XV oder unter synchroner Stickstoffabspaltung zum Nitron XVI führt.

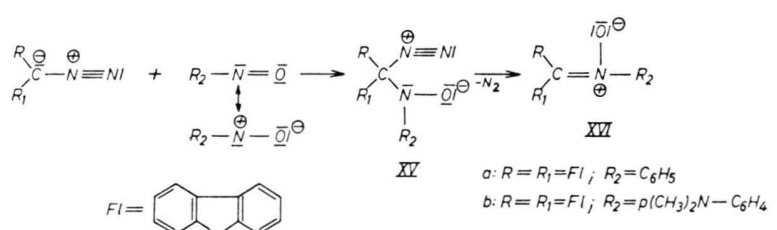

\begin{tabular}{|c|c|c|c|c|c|c|}
\hline \multirow[b]{2}{*}{ Diazoverbindung } & \multirow[b]{2}{*}{ Nitrosoverbindung } & \multirow{2}{*}{$\begin{array}{l}\text { Reaktions- } \\
\text { Temperatur } \\
\left({ }^{\circ} \mathrm{C}\right)\end{array}$} & \multirow{2}{*}{$\begin{array}{l}\text { Vergleich von } \\
\text { Eigenzerfall und } \\
\mathrm{N}_{2} \text {-Entwicklung } \\
\text { der Reaktion }{ }^{26}\end{array}$} & \multicolumn{2}{|c|}{ RGK der Reaktion } & \multirow{2}{*}{$\begin{array}{c}\text { RGK des } \\
\text { Eigenzerfalls } \\
{\left[\mathrm{min}^{-1}\right]}\end{array}$} \\
\hline & & & & $\begin{array}{l}{\left[\mathrm{min}^{-1}\right.} \\
\left.\times \mathrm{mol}^{-1}\right]\end{array}$ & {$\left[\mathrm{min}^{-1}\right]$} & \\
\hline $\begin{array}{l}\text { Diazofluoren } \\
\text { Diazofluoren }\end{array}$ & $\begin{array}{l}\text { Nitrosobenzol } \\
\text { p-Nitroso-dimethyl- }\end{array}$ & $\begin{array}{l}20 \\
80^{30 \mathrm{a}}\end{array}$ & $\begin{array}{l}(+) \\
(+)\end{array}$ & $\begin{array}{ll}\text { ca. } & 0,5 \\
& 0,18\end{array}$ & & $\begin{array}{c}29 \\
2 \cdot 10^{-4} 28\end{array}$ \\
\hline $\begin{array}{l}\text { Diazoessigsäure- } \\
\text { äthylester }\end{array}$ & $\begin{array}{l}\text { annin } \\
\text { Nitrosobenzol }\end{array}$ & 60 & $(+)$ & 29 & & 29 \\
\hline Benzoyl-diazomethan & Nitrosobenzol & 110 & $(+)$ & 29 & & 29 \\
\hline Azibenzil & Nitrosobenzol & $70^{30 \mathrm{~b}}$ & $(-)^{31}$ Abb. 6 & & 0,03 & 0,03 \\
\hline $\begin{array}{l}\text { Benzoyl-diazoessig- } \\
\text { säure-methylester }\end{array}$ & Nitrosobenzol & $105^{30 \mathrm{~b}}$ & $(-)^{31}$ & & 0,008 & 0,008 \\
\hline Diazodimedon & Nitrosobenzol & $110^{30 \mathrm{c}}$ & $(-)^{31}$ & & 0,01 & 0,01 \\
\hline
\end{tabular}

Tab. 3. Die Umsetzung von Diazoverbindungen mit Nitrosoverbindungen ${ }^{25}$.

30 a) Wegen der schlechten Löslichkeit von p-Nitroso-dimethylanilin in Xylol 2,0 mMol der Nitrosoverbindung und $2,0 \mathrm{mMol}$ Diazofluoren in 10,0 ml Xylol. b) $2,0 \mathrm{mMol}$ der Diazoverbindung und $2,0 \mathrm{mMol}$ Nitrosobenzol in $10,0 \mathrm{ml}$
Xylol. c) 1,0 mMol Diazodimedon und 1,0 mMol Nitrosobenzol in 10,0 ml Xylol.

${ }^{31}$ Bei der Reaktion entsteht neben Stickstoff auch Kohlendioxyd. 
Die Umsetzung von Alkyl- und Aryl-diazomethanen mit Nitrosoverbindungen zu Nitronen ist präparativ von Staudinger und Miescher ${ }^{32}$ untersucht worden. Benzoyl-diazoessigsäure-methylester und Diazo-malonsäureester reagieren nach Angabe der Autoren nicht, Diazoessigsäureester nur langsam.

Wir erhielten bei der schon bei $+20^{\circ}$ ablaufenden Umsetzung von Diazofluoren mit Nitrosobenzol das Nitron XVI a in 83-proz. Ausbeute. Die Umsetzung von Diazofluoren mit $p$-Nitroso-dimethylanilin, die allerdings erst bei $80^{\circ}$ mit gut meßbarer Geschwindigkeit abläuft, liefert das Nitron XVI b in 80-proz. Ausbeute. Diazoessigsäure-äthylester und Nitrosobenzol geben ein nicht destillierbares Öl. Die Umsetzung von Benzoyl-diazomethan mit Nitrosobenzol verläuft erst bei $110^{\circ}$, so daß der Eigenzerfall des Diazoketons bereits eine Rolle spielt. Dennoch zeigt der Vergleich von Eigenzerfall und Stickstoffentwicklung der Reaktion eine deutliche "Aktivierung" der Diazogruppierung und beweist damit den elektrophilen Angriff des Nitrosobenzols. Die Aufarbeitung lieferte nur harzige Schmieren.

Die kinetische Untersuchung der Umsetzung von Azibenzil, Benzoyl-diazoessigsäure-methylester und Diazodimedon kompliziert sich dadurch, daß außer Stickstoff auch Kohlendioxyd entwickelt wird. Durch getrennte Bestimmung der Gesamtgas- und der Kohlendioxyd-Entwicklung läßt sich die Stickstoffentwicklung jedoch wie beschrieben eliminieren (vgl. Abb. 6). Wir erhielten so Kurven der Stickstoffentwicklung, die mit denen des Eigenzerfalls der $\alpha$-Carbonyl-diazo-Verbindung identisch waren. Die Nucleophilie dieser Diazoverbindungen reicht also nicht mehr aus, um eine Reaktion mit der Nitrosoverbindung auszulösen, sondern es erfolgt primäre Bildung der entsprechenden Ketene, die nun, wie die $\mathrm{CO}_{2}$-Entwicklung zeigt, die Nitrosoverbindung zum 1.2-Oxazetidinon-(4)-Derivat XVII addieren, das unter den Reaktionsbedingungen $u$. a. in Kohlendioxyd zerfällt ${ }^{33}$.
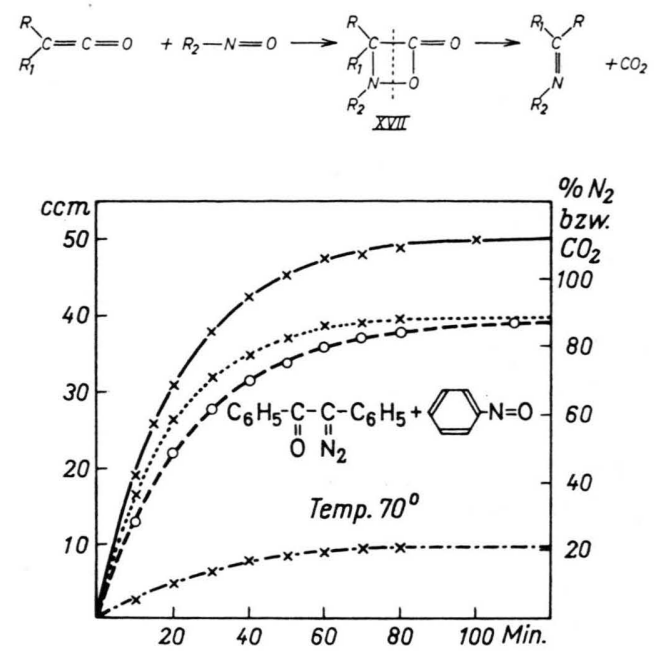

Abb. 6. Azibenzil mit Nitrosobenzol, - Gesamtgasentwicklung der Umsetzung von $2,0 \mathrm{mMol}$ Azibenzil mit 2,0 mMol Nitrosobenzol, - - - Eigenzerfall des Azibenzils, $\cdot-\cdot-\mathrm{CO}_{2}$-Entwicklung, $\cdots \mathrm{N}_{2}$-Entwicklung der Umsetzung ${ }^{34}$.

\section{Die Umsetzung von Diazoverbindungen mit $\mathrm{S} \mathrm{ch}$ if $\mathrm{f}$ schen Basen}

Wie Tab. 4 zeigt, sind bei der Umsetzung von Diazoverbindungen mit $\mathrm{S} \mathrm{ch}$ if $\mathrm{f}$ schen Basen mit Ausnahme der Umsetzung von Diazofluoren der Eigen-

\begin{tabular}{|c|c|c|c|c|c|}
\hline Diazoverbindung & Schiffsche Base & $\begin{array}{c}\text { Reaktions- } \\
\text { Temperatur } \\
{\left[{ }^{\circ} \mathrm{C}\right]}\end{array}$ & $\begin{array}{l}\text { Vergleich von } \\
\text { Eigenzerfall und } \\
\mathrm{N}_{2} \text {-Entwicklung } \\
\text { der Reaktion } 26\end{array}$ & $\begin{array}{c}\text { RGK der } \\
\text { Reaktion } \\
{\left[\mathrm{min}^{-1}\right]}\end{array}$ & $\begin{array}{l}\text { RGK des } \\
\text { Eigenzerfalls } \\
{\left[\min ^{-1}\right]}\end{array}$ \\
\hline Diazofluoren & Benzilidenanilin & 110 & 35 & & \\
\hline Azibenzil & Benzilidenanilin & 80 & $(-)$ & 0,2 & 0,2 \\
\hline Azibenzil & Benzophenonanil & 80 & $(-)$ & 0,2 & 0,2 \\
\hline Azibenzil & Cyclohexanonanil & 80 & $(-)$ & 0,2 & 0,2 \\
\hline $\begin{array}{l}\text { Benzoyl-diazoessigsäure- } \\
\text { methylester }\end{array}$ & Benzilidenanilin & 120 & $(-)$ & 0,07 & 0,04 \\
\hline $\begin{array}{l}\text { Benzoyl-diazoessigsäure- } \\
\text { methylester }\end{array}$ & Cyclohexanonanil & 120 & $(-)$ & 0,05 & 0,04 \\
\hline Diazodimedon & Benzilidenanilin & 120 & $(-)^{31}$ & 29 & \\
\hline
\end{tabular}

Tab. 4. Die Umsetzung von Diazoverbindungen mit $\mathrm{S} \mathrm{c} \mathrm{h}$ if $\mathrm{f}$ schen Basen ${ }^{25}$.

32 H. Staudinger u. K. Miescher, Helv. chim. acta 2, 554 [1919].

33 Vgl. dazu H. Staudinger u. S. Jelagin, Ber. dtsch. chem. Ges. 44, 365 [1911] sowie G. Kresze u. A. Trede, Tetrahedron [London] 19, 133 [1963].
34 Rechnerisch aus den Werten der Gesamtgas- und Kohlendioxyd-Entwicklung bestimmt.

35 Siehe dazu die Ausführungen auf S. 534. 
zerfall und die Geschwindigkeit der Stickstoffentwicklung der Reaktion identisch, d.h. daß diese Reaktionen als "Carben-Reaktionen“ ablaufen und damit Reaktionsprodukte der entsprechenden Ketene liefern müssen. In Übereinstimmung damit erhielten wir Derivate des Azetidinon-Systems XVIII:

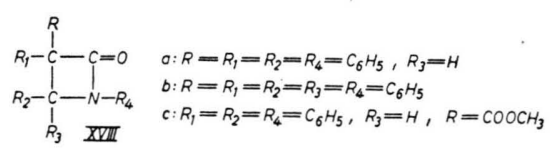

Aus Azibenzil und Benzilidenanilin XVIII a, aus Azibenzil und Benzophenonanil XVIII b und aus Benzoyl-diazoessigsäure-methylester und Benzilidenanilin XVIII c ${ }^{36}$.

Bei der Umsetzung von Diazofluoren mit Benzilidenanilin läuft die Stickstoffentwicklung der Reaktion zwar schneller als der Eigenzerfall, die Kurve zeigt jedoch einen unspezifischen Verlauf. Wir vermuten deshalb, daß hier ein Fall vorliegt, bei dem ein Ablauf nach Mechanismus C erfolgt. Dieser Befund steht in Übereinstimmung mit den Untersuchungen von Mustafa ${ }^{37}$, Buckley ${ }^{38}$ sowie Kadaba und Edwards ${ }^{39}$, die bei der Umsetzung von Diazomethan und Diphenyl-diazomethan mit Schiffschen Basen die Bildung der Triazoline XIX beobachteten, die beim Erwärmen unter Stickstoffabspaltung in die S chiff schen Basen und Zersetzungsprodukte der Diazoverbindungen zerfallen. In unserem Fall konnten wir nur das Olefin XX isolieren.
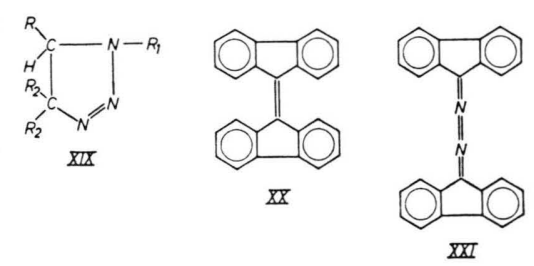

Die Umsetzung von Diazofluoren mit Cyanessigsäureester, $p$-Tolunitril und Nitromethan

Die Ergebnisse der Tab. 5 (vgl. auch Abb. 7) zeigen, daß die Umsetzung von Diazofluoren mit Cyanessigsäure-äthylester, $p$-Tolunitril und Nitromethan ohne Aktivierung der Diazoverbindung abläuft. Damit ist es also illusorisch im Reaktionsansatz nach Umsetzungsprodukten der Diazoverbindung selbst zu suchen. Dementsprechend lieferte die Aufarbeitung bei der Umsetzung mit Cyanessigsäure-äthylester bzw. Nitromethan das Ketazin XXI und mit $p$-Tolunitril das Olefin XX.

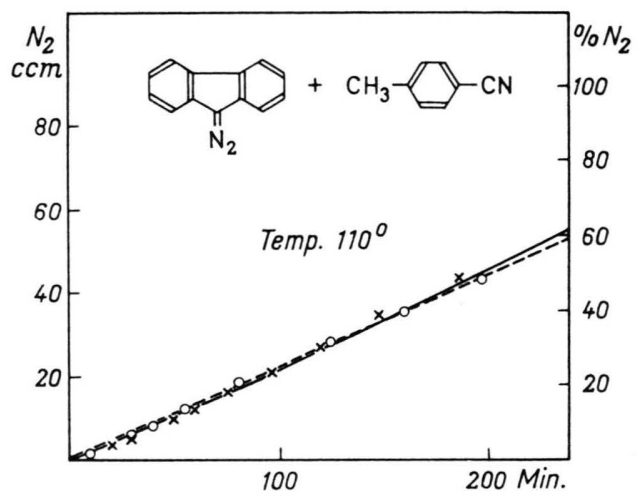

Abb. 7. Diazofluoren mit $p$-Tolunitril, $-x-x-$ Umsetzung ${ }^{25}$, $\circ--\circ$ Eigenzerfall des Diazofluorens.

\section{Experimentelles}

Biphenylen-N-phenyl-nitron (XVI a). - Zu 0,43 g $(4,0 \mathrm{mMol})$ Nitrosobenzol in $10 \mathrm{~cm}^{3}$ absol. Xylol werden $0,77 \mathrm{~g}(4,0 \mathrm{mMol})$ Diazofluoren gegeben. XVI a scheidet sich unter lebhafter Stickstoffentwicklung $a b$; Umkrist. aus Benzol/Petroläther (Sdp. $30-50^{\circ}$ ). Ausb. 83\% d. Th.; Schmp. $192-193^{\circ}$.

Biphenylen - $N$ - [p - dimethylamino - phenyl] - nitron

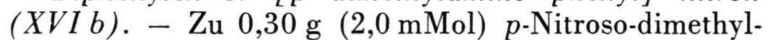
anilin in $10 \mathrm{~cm}^{3}$ absol. Xylol werden bei $80^{\circ} 0,38 \mathrm{~g}$ $(2,0 \mathrm{mMol})$ Diazofluoren gegeben. Umkrist. des Nitrons aus Benzol/Ligroin oder aus Isoamylalkohol.

\begin{tabular}{|l|c|c|c|}
\hline Reaktionskomponente & $\begin{array}{c}\text { Vergleich von Eigenzerfall } \\
\text { und N2-Entwicklung } \\
\text { der Reaktion }{ }^{26}\end{array}$ & $\begin{array}{c}R G K \text { der } \\
\text { Reaktion } \\
{\left[\mathrm{min}^{-1}\right]}\end{array}$ & $\begin{array}{c}R G K \text { des } \\
\text { Eigenzerfalls } \\
{\left[\mathrm{min}^{-1}\right]}\end{array}$ \\
\hline Cyanessigsäure-äthylester & $(-)$ & $4 \cdot 10^{-3}$ & $3 \cdot 10^{-3}$ \\
p-Tolunitril & $(-)$ Abb. 7 & $\begin{array}{l}3 \cdot 10^{-3} \\
4 \cdot 10^{-3}\end{array}$ & $\begin{array}{c}3 \cdot 10^{-3} \\
3 \cdot 10^{-3}\end{array}$ \\
\hline Nitromethan & $(-)$ & $4 \cdot 10^{-3}$ & \\
\hline
\end{tabular}

Tab. 5. Die Umsetzung von Diazofluoren mit Cyanessigsäure-äthylester, $p$-Tolunitril und Nitromethan ${ }^{25}$ bei $110^{\circ}$.

36 a) Die Struktur der bei der Umsetzung von Cyclohexanonanil mit Azibenzil oder Benzoyl-diazoessigsäure-methylester entstehenden Produkte konnte von uns nicht aufgeklärt werden. b) Die Bildung dieser Azetidinone wurde von W. Kirmse u. L. Horner, Chem. Ber. 89, 2759 [1956] bei der photochemischen Zersetzung von $\alpha$-Carbonyl-diazo-
Verbindungen in Gegenwart von $\mathrm{S} \mathrm{c}$ h if $\mathrm{f}$ schen Basen beobachtet.

37 A. Mustafa, J. chem. Soc. [London] 1949, 234.

38 G. D. Buckley, J. chem. Soc. [London] 1954, 1850.

39 P. K. Kadaba u. J. O. Edwards, J. org. Chemistry 26, 2331 [1961]. 
Ausb. $80 \%$ d. Th.; Schmp. $219^{\circ}$ (Isoamylalkohol), $208-209^{\circ}$ (Benzol/Ligroin mit etwas $\left.\mathrm{CHCl}_{3}\right)^{40}$.

Die Umsetzung von Diazofluoren mit Benzilidenani. lin. - 0,73 g (4,0 mMol) Benzilidenanilin und $0,77 \mathrm{~g}$ (4,0 mMol) Diazofluoren in $10 \mathrm{~cm}^{3}$ absol. Xylol werden auf $110^{\circ}$ erhitzt. Nach Beendigung der Gasentwicklung wird das Lösungsmittel i. Vak. abgezogen und das als Öl verbleibende $\alpha, \beta$-Bisdiphenylen-äthylen (XX) aus Chloroform/Methanol umkristallisiert. Ausb.: $35 \%$ d. Th.; Schmp. $187-188^{\circ}$.
Die Umsetzung von Azibenzil, Benzoyl-diazoessigsäure-methylester und Diazodimedon mit Benzilidenanilin, Benzophenonanil und Cyclohexanonanil. $\mathrm{Zu}$ einer Lösung von 4,0 mMol der $\mathrm{S} \mathrm{ch}$ if $\mathrm{f}$ schen Base in $10 \mathrm{~cm}^{3}$ absol. Xylol werden bei der in der Tabelle angegebenen Temperatur 4,0 mMol der Diazoverbindung gegeben. Nach Beendigung der Gasentwicklung wird das Lösungsmittel i. Vak. abgezogen und das als Rückstand verbleibende Azetidinon XVIII umkristallisiert (s. Tabelle 6).

\begin{tabular}{|c|c|c|c|c|}
\hline Diazoverbindung & Schiff sche Base & $\begin{array}{l}\text { Reaktions- } \\
\text { Temperatur } \\
{\left[{ }^{\circ} \mathrm{C}\right]}\end{array}$ & Umsetzungsprodukte & $\begin{array}{c}\text { Ausbeute } \\
(\% \text { d. Th. })^{36 b}\end{array}$ \\
\hline $\begin{array}{l}\text { Azibenzil } \\
\text { Azibenzil } \\
\text { Benzoyl-diazoessigsäure-methylester } \\
\text { Diazodimedon }\end{array}$ & $\begin{array}{l}\text { Benzilidenanilin } \\
\text { Benzophenonanil } \\
\text { Benzilidenanilin } \\
\text { Cyclohexanon- } \\
\text { anil }\end{array}$ & $\begin{array}{r}80 \\
80 \\
120 \\
120\end{array}$ & $\begin{array}{l}\text { Azetidinon XVIIIa } \\
\text { Azetidinon XVIIIb } \\
\text { Azetidinon XVIIIc } \\
\text { rotes Öl, nicht analysenrein } \\
\text { zu erhalten }\end{array}$ & $\begin{array}{l}16 \\
42 \\
36\end{array}$ \\
\hline
\end{tabular}

Tab. 6. Umsetzung von $a$-Carbonyl-diazo-Verbindungen mit $\mathrm{S} \mathrm{ch}$ if $\mathrm{f}$ schen Basen.

Umsetzung von Cyanessigsäure-äthylester, p-Tolunitril oder Nitromethan. Eine Lösung von 4,0 mMol Diazofluoren und 4,0 mMol Cyanessigsäureester, $p$-Tolunitril oder Nitromethan werden in $10 \mathrm{~cm}^{3}$ absol. Xylol auf $110^{\circ}$ erwärmt. Nach Beendigung der Gasentwicklung wird das Lösungsmittel i. Vak. abgezogen und der Rückstand aus Chloroform/Methanol umkristallisiert (Tab. 7).

Wir danken dem Fonds der Chemischen Industrie für die freundliche Gewährung von Forschungsbeihilfen.

40 Von E. Bergmann u. J. Hervey, Ber. dtsch. chem. Ges. 62, 893 [1929], wurde ein Schmp. von $223-224^{\circ}$, von F. Kröhnкe, Chem. Ber. 83, 253 [1950], von $201^{\circ}$ angegeben.

\begin{tabular}{|c|l|c|}
\hline Reaktionspartner & $\begin{array}{c}\text { Umsetzungs- } \\
\text { produkte }\end{array}$ & $\begin{array}{c}\text { Ausbeute } \\
{[\% \text { d. Th. }]}\end{array}$ \\
\hline $\begin{array}{c}\text { Cyanessigsäure- } \\
\text { äthylester }\end{array}$ & Ketazin XXI & 20 \\
$\begin{array}{c}p \text {-Tolunitril } \\
\text { Nitromethan }\end{array}$ & $\begin{array}{l}\text { Olefin XX } \\
\text { Ketazin XXI }\end{array}$ & $\mathbf{7 0}$ \\
\hline
\end{tabular}

Tab. 7. Zersetzung von Diazofluoren in Gegenwart von Cyanessigsäure-äthylester, $p$-Tolunitril oder Nitromethan bei $110^{\circ}$.

An dem von uns hergestellten Produkt beobachteten wir, daß der Schmp. von der Art des zur Umkristallisation verwandten Lösungsmittels abhängig ist. 